\title{
Astrocyte End-Feet in Germinal Matrix, Cerebral Cortex, and White Matter in Developing Infants
}

\author{
NADINE EL-KHOURY, ALEX BRAUN, FURONG HU, MAYA PANDEY, MAIKEN NEDERGAARD, \\ EDMUND F. LAGAMMA, AND PRAVEEN BALLABH
}

\begin{abstract}
Department of Pediatrics [N.E.-K., E.F.L., P.B.], Department of Pathology [A.B.], Department of Cell Biology and Anatomy [F.H., M.P., P.B.], Westchester Medical Center, New York Medical College, Valhalla, New York 10595; Department of Neurosurgery [M.N.], Center of Aging and Developmental Biology, University of Rochester, Rochester, New York 14642
\end{abstract}

\begin{abstract}
Astrocyte end-feet ensheathe blood vessels in the brain and are believed to provide structural integrity to the cerebral vasculature. We sought to determine in developing infants whether the coverage of blood vessels by astrocyte end-feet is decreased in germinal matrix (GM) compared with cerebral cortex and white matter (WM), which may cause fragility of the GM vasculature. Therefore, we evaluated the perivascular coverage by astrocyte endfeet in these areas. We double-labeled the brain sections with astroglial markers [glial fibrillary acidic protein (GFAP), aquaporin-4 (AQP4), and S-100 $\beta$ ] and a vascular marker, laminin. Perivascular coverage by GFAP + astrocyte end-feet increased consistently as a function of gestational age (GA) in cortex and WM from 19 to $40 \mathrm{wk}$. Compared with GFAP, AQP4+ astrocyte end-feet developed at an earlier GA, ensheathing about $63 \%$ of blood vessels for $23-40 \mathrm{wk}$ in cortex, WM, and GM. Coverage by GFAP+ perivascular end-feet was decreased in GM compared with cortex and WM from 23 to 34 wk. There was no difference in the coverage by AQP4+ end-feet among the three areas in these infants. The expression of AQP4, a water channel molecule, in the astrocyte end-feet was not significantly different between premature and mature infants, suggesting similar risk of brain edema in preterm and term infants in pathologic conditions. More importantly, the lesser degree of GFAP expression in astrocyte end-feet of GM compared with cortex and WM may reflect a cytoskeletal structural difference that contributes to the fragility of GM vasculature and propensity to hemorrhage. (Pediatr Res 59: 673-679, 2006)
\end{abstract}

$\mathrm{G}$ erminal matrix hemorrhage (GMH)-intraventricular hemorrhage (IVH) is a major problem of premature infants because a large number of these babies develop cerebral palsy, hydrocephalus, and mental retardation $(1,2)$. The GM located in the thalamostriate groove beneath the ependyma is an undifferentiated collection of glial and neuronal precursor cells with rich vascular network, present until late gestation. Premature infants primarily bleed into the GM and not into the cortex or WM, suggesting that blood vessels in the GM are fragile. Astrocyte end-feet tightly ensheathe blood vessels in the brain to form a BBB and are viewed as providers of structural integrity to the blood vessels (1). In this study, we

Received August 16, 2005; accepted January 10, 2006.

Correspondence: Praveen Ballabh, M.D., NICU, 2nd Floor, Maria Fareri Children's Hospital, Valhalla, NY 10585; e-mail: Pballabh@msn.com

Supported by the United Cerebral Palsy Educational and Research Foundation and The Hirsch, Foundation Inc.

DOI: $10.1203 / 01 . p d r .0000214975 .85311 .9 c$ asked whether coverage of the blood vessels by astrocyte end-feet is decreased in the germinal matrix compared with cortex and WM, which may cause fragility of the GM vasculature.

The pathogenesis of GM vascular fragility and its vulnerability to hemorrhage is not well understood. We have shown previously that the increased vascularity and the relatively circular shape of blood vessels in the GM compared with the cerebral cortex and WM may play a role in GMH of premature infants (3). Further, our investigation on endothelial TJ molecules of the BBB has also shown that the primary TJ molecules including claudin-5, occludin, and junction adhesion molecule-1 are not decreased in GM compared with cortex and WM and, thus, are unlikely to play a role in GM fragility (4). Therefore, an evaluation of astrocyte end-feet, another component of the BBB, may be critical to understanding the reason of GM fragility.

Development of the BBB in the GM has been studied in baboon and beagle pup models at the developmental stage, during which premature infants develop $\operatorname{GMH}(5,6)$. In human fetuses, it has been observed that perivascular coverage by astrocyte end-feet in cortical plate is greater at $18 \mathrm{wk}$ compared with 12 wk GA (7). In adult humans, electron microscopy revealed that $80 \%$ of the vascular endothelium-pericyte layer is invested by astrocyte end-feet (8). However, there is no systematic human data in the literature on blood vessel coverage by astrocyte end-feet in GM compared with cortex and WM in premature infants. Therefore, we have sought to determine the perivascular coverage by astrocyte end-feet in the GM compared with cortex and WM from 16 to $40 \mathrm{wk}$ GA. In addition, we have examined whether there is a correlation between extent of perivascular coverage by glial end-feet and dimensions of the blood vessels including length, breadth, and perimeter.

For this purpose, we have used three well-known markers of astrocyte end-feet: GFAP, AQP4, and S-100 $\beta$. GFAP, an intermediate filament protein, is exclusively expressed in glial

Abbreviations: AQP4, aquaporin-4; BBB, blood brain barrier; GA, gestational age; GFAP, glial fibrillary acidic protein; GM, germinal matrix; TJ, tight junction; WM, white matter 
cells (9). AQP4 expression is highly polarized and most immunoreactivity is present in the astrocyte end-feet in direct contact with capillaries $(10,11)$. Finally, S- $100 \beta$, a soluble calcium-binding protein, is found mainly in astroglial cells and has been widely used as a specific marker of glial cells (12).

\section{MATERIALS AND METHODS}

The Institutional Review Board at New York Medical College, Valhalla, NY, approved the use of human autopsy materials for this study. Study material included autopsy brain samples from premature infants $23-40 \mathrm{wk}$ and spontaneous abortuses 16-22 wk. Only autopsy samples of $<18-\mathrm{h}$ postmortem interval were used. Infants with major congenital anomalies, chromosomal defects, culture-proven sepsis, meningitis, or hypoxic-ischemic encephalopathy and infants receiving extracorporeal membrane oxygenator treatment were excluded. Informed consent was obtained for autopsy.

Tissue collection and processing. Brain samples were obtained as 1-2$\mathrm{mm}$-thick coronal blocks by sectioning through frontal cortex (cortical plate), frontal WM (embryonic intermediate layer), and GM in the region of the thalamostriate groove and at the level of foramen of Monro. Samples were fixed in $4 \%$ paraformaldehyde in $0.01 \mathrm{M}$ PBS for $18 \mathrm{~h}$ then cryoprotected by immersion into $20 \%$ sucrose in $0.01 \mathrm{M}$ PBS for $24 \mathrm{~h}$. Frozen coronal blocks were cut into $20-\mu \mathrm{m}$ sections.

Immunohistochemistry. Primary antibodies included rabbit polyclonal aquaporin-4 (Santa Cruz Biochemicals, Santa Cruz, CA; 1:200), mouse monoclonal GFAP (Sigma Chemical Co., St. Louis, MO; 1:500), rabbit polyclonal anti-human S-100 $\beta$ (DAKO, Carpinteria, CA; 1:200), mouse monoclonal laminin (Sigma Chemical Co.; 1:1000), and rabbit polyclonal laminin (Sigma Chemical Co.; 1:1000). Secondary antibodies were Cy-5 conjugate goat-anti-mouse, rhodamine conjugate goat-anti-rabbit, and cy-5 conjugate goat-anti-rabbit (Jackson Immunoresearch, West Grove, PA). To co-localize the expression of aquaporin-4, GFAP, and S- $100 \beta$ in relation to vascular basal lamina, we double-labeled the sections using a combination of rabbit polyclonal AQP4 or S-100 $\beta$ with mouse monoclonal laminin and mouse monoclonal GFAP with rabbit polyclonal laminin. Sections were initially incubated with 5\% normal goat serum and 0.2\% TritonX-100 in PBS for $2 \mathrm{~h}$ at room temperature, then incubated overnight at $4^{\circ} \mathrm{C}$ with primary antibodies diluted in $0.2 \%$ Triton X-100 in PBS. Finally, after washes in PBS, sections were incubated in secondary antibody for $2 \mathrm{~h}$ at room temperature. Subsequently, sections were mounted with Slow-Fade Light-Antifade (Molecular Probes, Eugene, OR). Controls were made by replacing primary antibodies with $0.2 \%$ Triton X-100 in PBS.

Confocal microscopy and image analysis. For each brain, three to five coronal sections were selected as every 10th section. For each section, five images were acquired from each area-GM, cortex, and WM. We acquired a total of 45-75 images (3-5 sections $\times 3$ areas $\times 5$ images) per brain for each astrocyte marker, yielding 2754, 2832, and 1,732 blood vessels with GFAP, AQP4, and S- $100 \beta$ immunostaining, respectively. Sections were examined under confocal laser-scanning microscope (MRC-1024ES; Bio-Rad, Hemel Hempstead, UK) using a $60 \times$ objective. Two-dimensional images were acquired and analyzed for the overlapping of the perimeter of blood vessels and astrocyte end-feet. AQP4, GFAP, or S-100 $\beta$ labeled the end-feet and laminin stained the vascular basal lamina. Because sections were double labeled, blood vessels stained with anti-laminin were seen in blue (cy5 florescent probe) and astrocytes stained with GFAP, AQP4, or S-100 $\beta$ in red (rhodamine florescent probe). Metamorph version-6.1 software (Universal Imaging Corporation, Downington, PA) was used for analysis. The two sources of image, astrocyte (red) and laminin (blue), were displayed on the Metamorph screen. Both sources of images were thresholded. "Region tool" in the software was then used to define and select the outer margin of the blood vessel under consideration. The software then measured this delineated area in pixels and calculated the percentage overlap (co-localization) of red (astrocyte) over blue signal (blood vessel). Thus, each blood vessel margin in every image was individually selected to evaluate overlap with the astrocyte end-feet. The blood vessels dimensions were measured separately in the laminin immunostained images to assess possible correlation between perivascular coverage and blood vessel dimensions.

Length, breadth, and perimeter of blood vessels were defined as follows: length $=$ span of the longest chord through blood vessel; breadth $=$ caliber width of object perpendicular to the longest chord; perimeter $=$ distance around edge of blood vessel measuring from points of each pixel that defines the border.
Statistics and analysis. Percentage perivascular coverage by AQP4, GFAP, and S-100 $\beta+$ end-feet was studied as a function of GA. Infants were stratified into five GA categories based on viability and maturity: 16-18, 19-22, 23-26, 27-34, and 35-40 wk. Mean perivascular coverage was compared between GM, cortex, and WM and across GA categories. We used Mann-Whitney test for paired comparisons and ANOVA for comparisons across GA categories. A value of $p<0.05$ was considered significant.

To study the relationship between percentage perivascular coverage and blood vessel dimensions, we performed linear regression analysis for the independent variable, percentage co-localization, and dependent variables, length, breadth, and perimeter, and calculated $R^{2}$.

\section{RESULTS}

Characteristics of subjects are depicted in Table 1. The subjects were divided into five GA categories, including previable [16-19 $(n=4)$ and 20-22 $(n=3) \mathrm{wk}]$ and viable [23-26 $(n=5), 27-34(n=4)$ and 35-40 $(n=3) \mathrm{wk}]$.

Immunoreactivity of GFAP, AQP-4, and S-100ß. Sections stained without primary antibodies or without secondary antibodies (negative control) showed total absence of labeling (data not shown) (Fig. 1). GFAP, an intermediate filament protein, is exclusively expressed in astrocytes (13). GFAP immunolabeling of $20-\mu$ sections from term infant brain showed numerous stellate astrocytes with multiple radiating processes, some extending to the wall of nearby vessels as

Table 1. Characteristics of the fetuses and infants

\begin{tabular}{|c|c|c|c|}
\hline $\begin{array}{l}\text { Postconceptional } \\
\text { age (wk) }\end{array}$ & Sex & $\begin{array}{l}\text { Birth } \\
\text { weight } \\
(\mathrm{kg})\end{array}$ & $\begin{array}{l}\text { Cause of death/ } \\
\text { spontaneous termination }\end{array}$ \\
\hline 16 & Male & & Cervical incompetence \\
\hline 16 & Female & & Spontaneous termination \\
\hline 17 & Male & & Spontaneous termination \\
\hline 18 & Male & & Spontaneous termination \\
\hline 19 & Female & & Cervical incompetence \\
\hline 20 & Male & 0.24 & Preterm labor \\
\hline 22 & Female & 0.35 & Twins in preterm labor \\
\hline $23 *$ & Female & 0.59 & $\begin{array}{l}\text { Respiratory failure } \\
\qquad(\text { died postnatal d 1) }\end{array}$ \\
\hline $23 \dagger$ & Female & 0.68 & $\begin{array}{l}\text { Respiratory failure } \\
\quad(\text { died postnatal d 2) }\end{array}$ \\
\hline 24 & Female & 0.59 & $\begin{array}{l}\text { Pneumothorax, respiratory } \\
\text { failure }\end{array}$ \\
\hline $24 \dagger$ & Male & 0.63 & $\begin{array}{l}\text { Respiratory failure } \\
\text { (died postnatal d 3) }\end{array}$ \\
\hline $26 \ddagger$ & Male & 0.62 & $\begin{array}{l}\text { Kidney failure } \\
\qquad(\text { died postnatal d } 3)\end{array}$ \\
\hline $27 \dagger$ & Female & 1.02 & $\begin{array}{l}\text { Respiratory failure } \\
\quad(\text { died postnatal d 2) }\end{array}$ \\
\hline $29 \dagger$ & Male & 1.12 & $\begin{array}{l}\text { Necrotizing enterocolitis } \\
\text { (born } 24 \mathrm{wk} \text { GA, } \\
\text { died } 4 \mathrm{wk} \text { ) }\end{array}$ \\
\hline 31 & Male & 1.34 & Unproven sepsis \\
\hline 32 & Male & 1.4 & $\begin{array}{l}\text { Perforated ileum } \\
\text { (born } 31 \text { wk GA, } \\
\text { died postnatal d 5) }\end{array}$ \\
\hline 37 & Female & 2.64 & $\begin{array}{l}\text { Diaphragmatic hernia } \\
\quad \text { (died } 22 \mathrm{~h})\end{array}$ \\
\hline 40 & Female & 3.4 & $\begin{array}{l}\text { Cardiomyopathy } \\
\text { (died } 2 \text { wk) }\end{array}$ \\
\hline 40 & Female & 3.5 & Stillbirth \\
\hline
\end{tabular}

* Grade I-II IVH.

$\dagger$ Grade III IVH. None of the infants had grade IV IVH.

$\uparrow$ All preterm infants except one were exposed to prenatal steroids. 


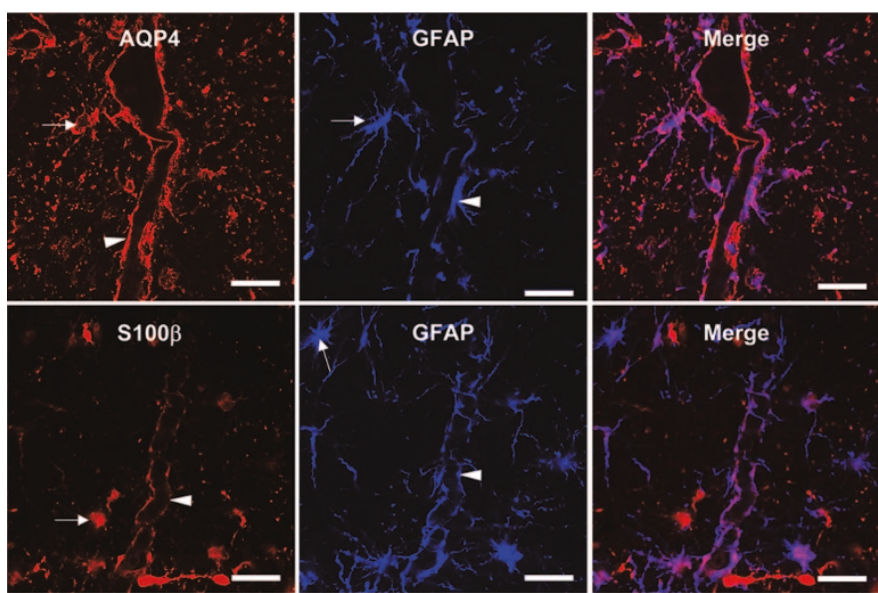

Figure 1. Double immuno-labeling of brain sections from term infants. (Upper panel) Aquaporin-4 with GFAP: Coronal sections of the brain stained with anti-GFAP (blue) and anti-AQP4 (red) antibodies. Anti-GFAP stains stellate astrocytes (arrow), processes, and end-feet (arrowhead) equally, whereas anti-AQP4 labels astrocyte end-feet (arrowhead) almost continuously around the blood vessels. AQP4 immunoreactivity is also seen in the membrane of cell bodies of the astrocyte (arrow). The merge image shows remarkable co-localization of GFAP and AQP4, but differences include that AQP4 labels predominantly the end-feet and GFAP stains cell bodies, processes, and end-feet equally. (Lower panel) GFAP with S-100 $\beta$ : Coronal sections of the brain stained with anti-GFAP (blue) and anti-S100 $\beta$ (red) antibodies. Compared with GFAP immunostaining, S- $100 \beta$ antibody intensely stains the cell bodies of astrocyte and end-feet whereas labeling of fine astrocyte processes is weak. Scale bar $=25 \mu$.

end-feet. Aquaporins are water channel proteins, expressed in the cell membrane, and AQP4, in particular, is predominantly expressed in high concentration in the astrocyte end-feet $(10,14)$. AQP4 immunostaining of brain sections from term infants revealed intense staining of astrocyte end-feet almost entirely outlining the blood vessel. It also stained the plasma membrane of the astrocyte cell body, but not the cytoplasm. Immunoreactivity of astrocyte processes was noted in places. Immunostaining of $\mathrm{S}-100 \beta$, which is a cytosolic calciumbinding protein (12), revealed intensely stained astrocyte cell bodies and end-feet but weakly stained processes.

Comparing immunoreactivities for GFAP with AQP4 and S-100 $\beta$ in term infant brains revealed remarkable colocalization of all three antibodies, but there were differences. Anti-GFAP equally stained the cell body, processes, and end-feet of astrocytes. In contrast, anti-AQP4 labeled astrocyte end-feet and membrane of the cell body with only patchy staining of processes. S- $100 \beta$ antibody intensely stained the cell body and end-feet of astrocytes but labeled few processes.

Astrocyte end-feet in fetuses. Figure 2 depicts extensive $\mathrm{GFAP}+$ radial glial processes in cortex, WM, and GM, with only few astrocyte bodies and end-feet. In contrast, AQP4+ astrocyte bodies, processes, and end-feet were scarce in cortex and WM, whereas relatively well-developed end-feet and glia limitans were seen in GM. S- $100 \beta+$ astrocyte cell bodies with few thin processes and rare end-feet are seen in cortex, WM, and GM.

Astrocyte end-feet in premature and mature infants. In premature infants, GFAP labels astrocyte cell bodies, processes, and end-feet extensively in cortex and WM (Figs.

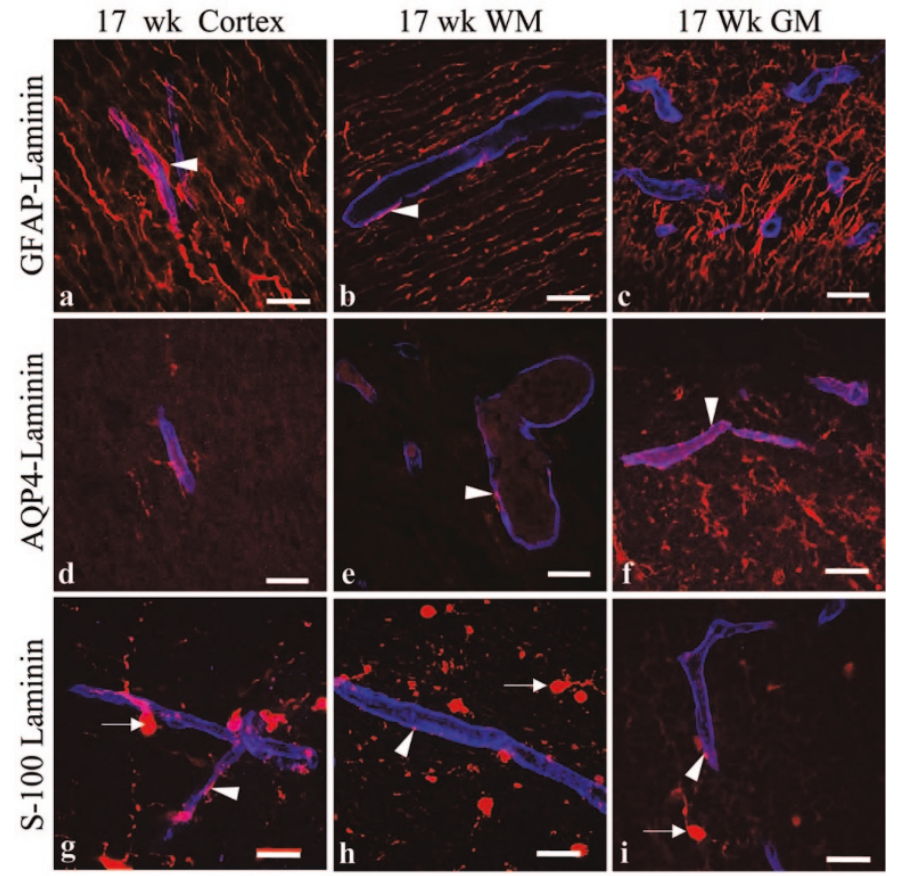

Figure 2. GFAP, AQP4, and S-100 $\beta$ (red) with laminin (blue) immunostaining for cortex, WM, and GM in 17-wk fetus. $(a-c)$ GFAP+ radial glial processes are extensive in cortex, WM, and GM, with only slight staining of end-feet (arrowhead). Radial glial fibers are closely packed around blood vessels. $(d-f) \mathrm{AQP} 4+$ astrocyte bodies and processes are scarce in cortex and WM. However, developed astrocyte end-feet covering blood vessels (arrowheads) and glia limitans are seen in GM. ( $g-i)$ S-100 $\beta$ intensely stains astrocyte cell bodies (arrow), with few processes in GM, cortex, and WM. End-feet (arrowhead) are few and barely covering blood vessels. Scale bar $=$ $25 \mu$.

3-5). However, in GM, GFAP labels radial glia and fibrous/ intermediate astrocytes with only few processes and end-feet. With advancing GA, GFAP+ end-feet coverage increased, with more mature-looking astrocytes in WM and cortex. Unlike GFAP, AQP4 staining showed strongly stained endfeet in cortex, WM, and GM. AQP4 also stained plasma membrane of the cell body, ependyma-attached radial glia, and ependyma. S- $100 \beta$ labeling showed intense staining of astrocyte cell bodies and ependyma-attached radial glia but only few thin processes and end-feet. Perivascular coverage by $\mathrm{S}-100 \beta+$ end-feet appears decreased in GM compared with cortex and WM.

In mature infants, GFAP stains stellate astrocytes, processes, and end-feet widely covering blood vessels in cortex and WM. AQP4 stains end-feet intensely in cortex and WM, with only few processes. S-100 $\beta$ labels end-feet sparsely covering blood vessels, with intense staining of astrocyte cell bodies.

Quantification of perivascular coverage by GFAP+ endfeet. GFAP + end-feet coverage increased consistently with GA in cortex and WM from 19 to $40 \mathrm{wk}(p<0.001)$ (Fig. $6 a)$. In contrast, in GM, it increased only between 19-22 and 23-26 wk $(p<0.05)$. There was no significant difference in GFAP+ end-feet coverage among GM, cortex, and WM for fetuses 16-22 wk, but there was decreased coverage in GM compared with cortex and WM in premature infants, 


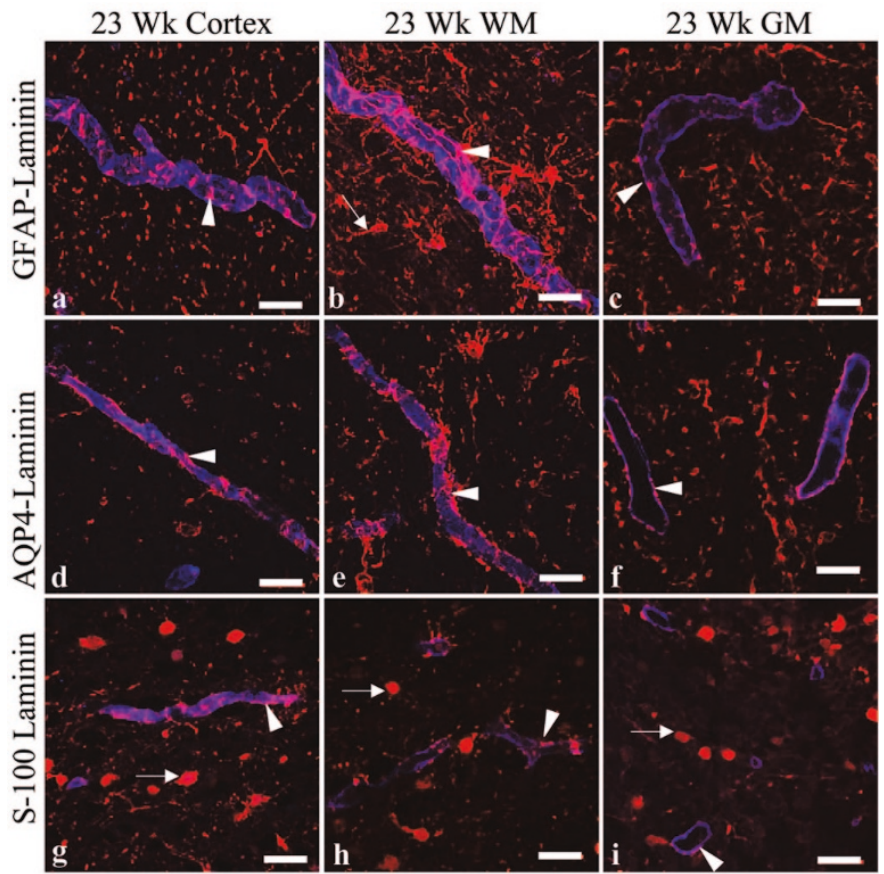

Figure 3. GFAP, AQP4, and $\mathrm{S}-100 \beta$ (red) with laminin (blue) immunostaining for cortex, WM, and GM in 23-wk premature infant. (a-c) GFAP+ astrocyte cell bodies, processes, and end-feet (arrowhead) are extensively covering blood vessels in cortex and WM. Stellate-shaped astrocytes (arrow) are seen more frequently in WM compared with cortex. End-feet are fewer and look immature in GM compared with cortex and WM. $(d-f)$, Unlike GFAP, AQP4+ end-feet (arrowhead) are strongly stained in GM ensheathing blood vessels, as in cortex and WM. $(g-i)$ Astrocyte cell bodies (arrow) with few processes and end-feet are intensely stained for S-100 $\beta$ in cortex and WM and even fewer end-feet in GM. Scale bar $=25 \mu$.

23-34 wk. Percentage perivascular coverage was about $61 \%$ in term infants, similar between cortex and WM.

Quantification of perivascular coverage by AQP4+ endfeet. AQP4+ end-feet coverage in GM increased significantly from $1.3 \pm 0.2 \%$ (mean $\pm \mathrm{SEM}$ ) at $16 \mathrm{wk}$ (not shown in graph) to $53 \pm 2.4 \%$ at $18-19 \mathrm{wk}$ and $63.7 \pm 1.9 \%$ at $20-22$ wk $(p<0.001)$, then plateaued (Fig. 6b). In cortex and $\mathrm{WM}$, it increased consistently from $<5 \%$ at $16-18$ wk to about $63 \%$ by $23-26$ wk $(p<0.001)$ then plateaued. Percentage coverage was significantly greater in GM than cortex and WM in fetuses, $16-18$ and $19-22 \mathrm{wk}(p<0.001)$. However, there was no significant difference in premature infants (23-26 and 27-34 wk). In term infants, coverage was greater in WM than in cortex $(72.4 \pm 1.2$ versus $61.2 \pm 1.4 ; p<0.001)$.

GFAP-negative end-feet. GFAP + end-feet were significantly decreased in GM $(p<0.001)$ and were about half of AQP4+ end-feet for 19-40 wk. Likewise, GFAP+ end-feet were significantly fewer than AQP4+ end-feet in cortex and WM for $19-40 \mathrm{wk}(p<0.05)$. This implies that about $50 \%$ of end-feet in GM are GFAP-negative and some end-feet are also GFAP-negative in cortex and WM.

Quantification of perivascular coverage of $S-100 \beta+$ endfeet. In GM, S-100 $\beta+$ perivascular coverage increased between 16-18 and 19-22 wk ( $p<0.05$ ), then plateaued (Fig. $6 c)$. However, in cortex and WM, coverage increased with GA 19-40 wk $(p<0.001)$, and no significant change was noted between $16-18$ and $19-22$ wk. S-100 $\beta+$ coverage was

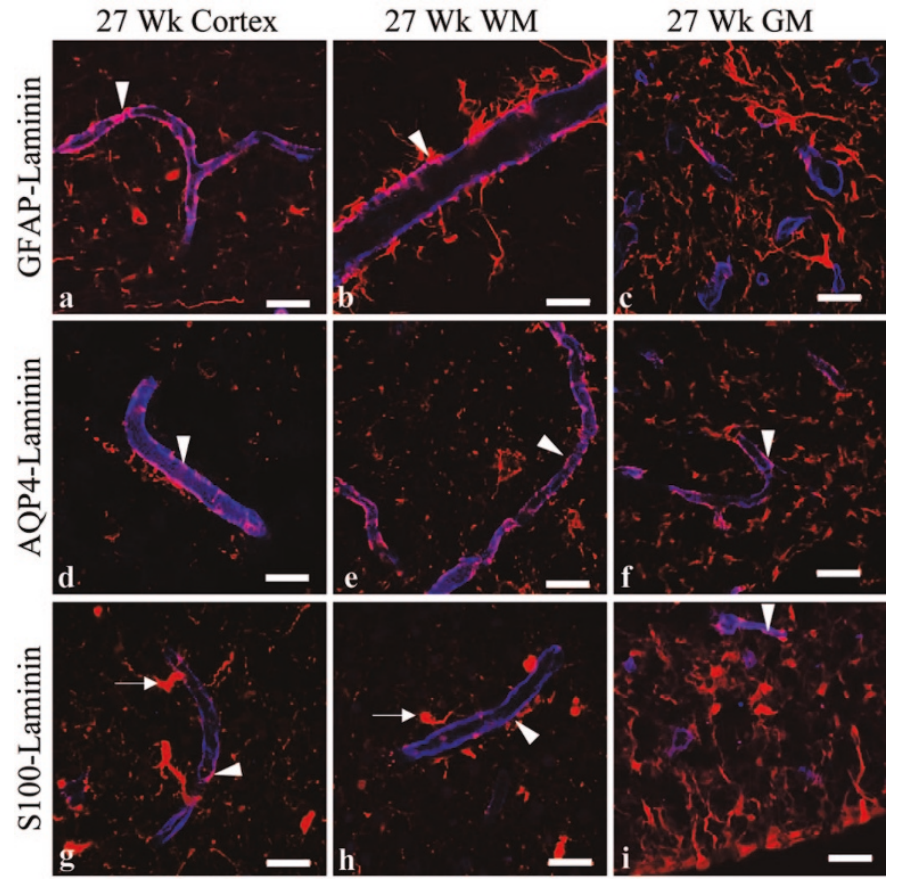

Figure 4. GFAP, AQP4, and S-100 $\beta$ (red) with laminin (blue) immunostaining for cortex, WM, and GM in 27 -wk premature infant. $(a-c)$ GFAP+ end-feet staining (arrowhead) is more extensive compared with $23 \mathrm{wk}$, along with cell bodies and processes in cortex and WM. However, GM shows only few end-feet with extensive network of glial fibers. $(d-f), \mathrm{AQP} 4+$ end-feet (arrowhead) are clearly covering blood vessels. ( $g-i)$ S- $100 \beta$ staining shows cell bodies (arrow) and few processes with end-feet covering sparsely blood vessels in cortex and WM. GM shows S-100 $\beta+$ ependyma-attached radial glia with few end-feet (arrowhead) covering blood vessels. Scale bar $=25 \mu$.

decreased in GM compared with cortex and WM for premature infants $23-34 \mathrm{wk}(p<0.001)$. In term infants, coverage was about $35 \%$, similar between cortex and WM.

Correlation between percent coverage and vessel dimensions. Linear regression analysis was performed. $R^{2}$ values approached zero for all three dependent variables for each marker, indicating no definite relationship between blood vessel dimensions and their coverage by astrocyte end-feet.

\section{DISCUSSION}

The present study evaluated perivascular coverage by astrocyte end-feet in GM, cortex, and WM in fetuses and premature infants $16-40 \mathrm{wk}$. We found that coverage by GFAP+ end-feet was decreased in GM compared with cortex and WM in premature infants 23-34 wk. However, there was no significant difference in perivascular coverage by AQP4+ end-feet in GM, cortex, and WM among these subjects.

We used three immunohistochemical markers, GFAP, AQP4, and S-100 $\beta$, to identify astrocyte end-feet and laminin to label vascular basement membrane. These markers have been widely used as specific glial and vascular markers $(3,12$, $13,15,16)$. We observed consistent and strong immunohistochemical staining with minimal background using these antibodies in coronal sections of the brain tissues. We did not use vimentin (type-III intermediate filament) as one of the immunohistochemical markers for astrocytes because it is not spe- 

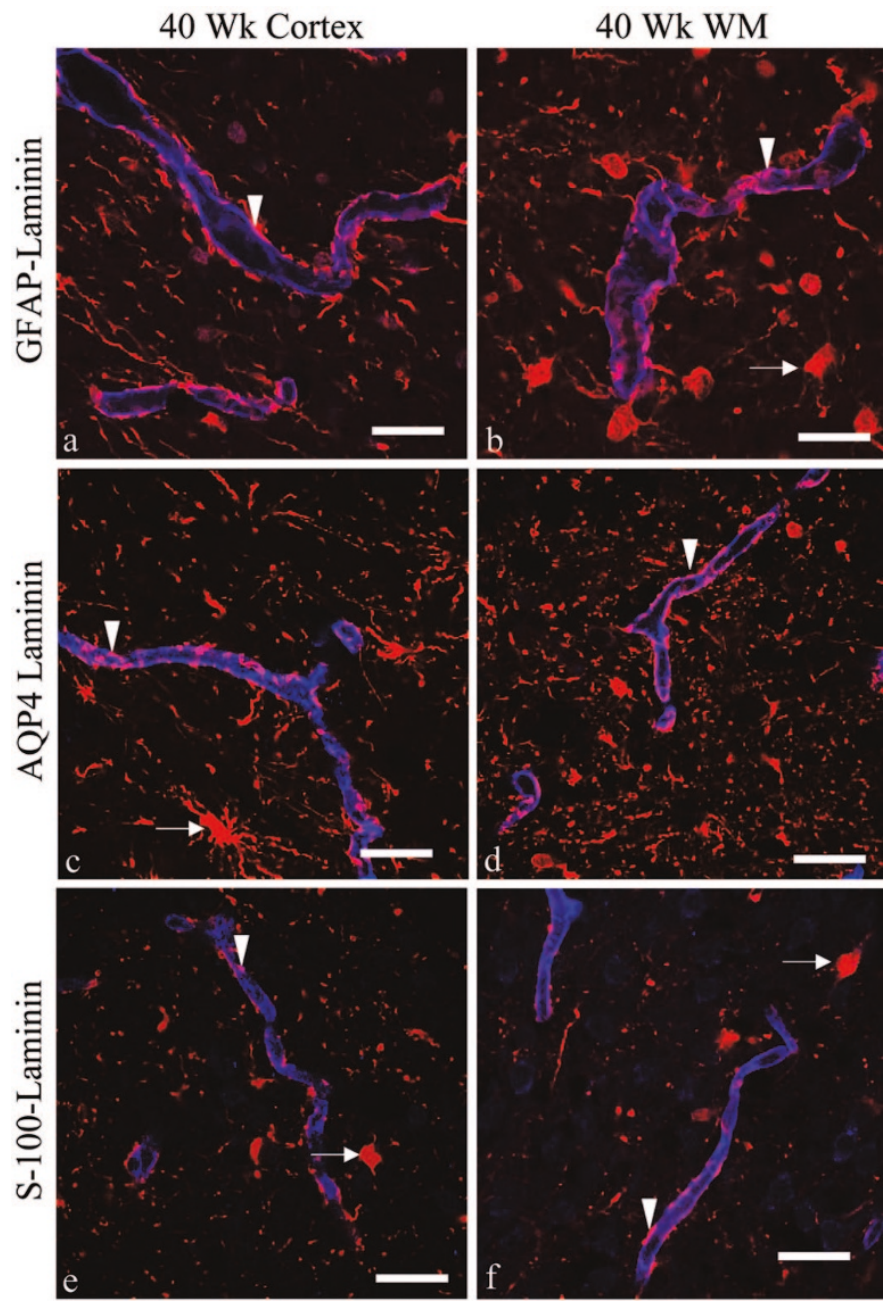

Figure 5. GFAP, AQP4, and S-100 $\beta$ (red) with laminin (blue) immunostaining for cortex, and WM in 40-wk infant. $(a, b)$ GFAP stains the stellate astrocytes (arrow), processes, and end-feet (arrowhead) covering blood vessels widely in cortex and WM. $(c, d) \mathrm{AQP} 4+$ end-feet are intensely stained, covering blood vessels in cortex and WM. AQP4 also stains few astrocyte processes. (e, $f$ ) S-100 $\beta$ labeling shows end-feet (arrowhead) sparsely covering blood vessels with intense staining of astrocyte cell bodies (arrow). Scale bar $=25 \mu$.

cific for astrocytes and is also expressed by endothelial cells, pericytes, and leukocytes (cells of mesenchymal origin) (17). We used confocal microscopy to acquire 45-75 sets of twodimensional images per brain for each glial marker in 19 subjects 16-40 wk GA. Thus, we analyzed a large number of high-resolution images to evaluate astrocyte end-feet in the brain of fetuses and premature and mature infants. In describing our observations, we used the term WM synonymously with the intermediate zone embryonic WM and cortex for the cortical plate for subjects 16-40 wk GA for the sake of simplicity and uniformity of presentation.

The most important and novel observation made in this study was that perivascular coverage by GFAP + end-feet was decreased in GM compared with cortex and WM for premature infants 23-34 wk GA, whereas there was no difference in coverage by $\mathrm{AQP} 4+$ astrocyte end-feet among GM, cortex, and WM for these infants. This implies that astrocyte end-feet are formed in GM as in cortex and WM, but are developmen- tally distinct with relative lack of GFAP expression. They also lack S-100 $\beta$, but GFAP is particularly important because it is a major cytoskeletal protein forming the intermediate filament of astrocytes. Several lines of evidence suggest that GFAP functions by providing shape and mechanical strength to astrocytes, including their end-feet. First, studies on GFAP deficient mice have revealed that their BBB is structurally and functionally impaired (18). Second, morphologic and metabolic studies indicate that an increase in GFAP accompanies differentiation of polygonal astroblasts into stellate astrocytes with multiple processes, which appear to provide structural integrity to astrocyte, processes, and end-feet $(19,20)$. Third, a biochemical hallmark of gliosis is up-regulation of GFAP, which constitutes the structural support of intermediate filament in differentiated astrocytes (21). Fourth, effects of steroid on GFAP in the GM are unknown, but corticosteroids upregulate GFAP in vitro $(22,23)$; and prenatal steroids are known to decrease the incidence of IVH (24). Therefore, it is plausible that a decrease in GFAP + expression in astrocytes of GM (compared with cortex or WM) may cause the fragility of the GM vasculature and its increased propensity for hemorrhage.

It has been reported that GFAP + glial cells appear and mature in different areas of the CNS at varied periods of gestation. GFAP staining of the brain of fetal baboon of $100 \mathrm{~d}$ GA revealed no GFAP+ astrocytes or processes in the GM, whereas stellate-shaped astrocytes and perivascular end-feet were observed in the WM and cortex (5). In humans, GFAP+ glial cells start at $9 \mathrm{wk}$ in the spinal cord, $14 \mathrm{wk}$ in internal capsule and thalamus, and 14-19 wk in the ependyma of the frontal cortex $(9,25-27)$. GFAP+ end-feet in cerebral hemisphere and brain stem are noted from $14 \mathrm{wk}$ versus $17 \mathrm{wk}$ in GM (9). Consistent with these findings, we also observed few GFAP + end-feet and glial fibers in GM at 16-17 wk. There is no systematic data on the quantification of GFAP+ astrocyte end-feet in premature infants in GM compared with cortex and WM, and this is the first report on decreased expression of GFAP in astrocyte end-feet of GM compared with cortex and WM.

Another key finding of the present study is the first demonstration of the expression of water channel molecule, $\mathrm{AQP} 4$, in the BBB of GM, cortex, and WM of fetuses and premature and mature infants. We observed that vascular coverage by AQP4+ end-feet in GM increased from $1.3 \%$ at $16 \mathrm{wk}$ to $53 \%$ at $18-19 \mathrm{wk}$ and $63.7 \%$ at $20-22 \mathrm{wk}$ GA, followed by plateauing, whereas in cortex and WM, it increased from $<5 \%$ at $16-18$ wk to $63 \%$ by $23-26$ wk GA, followed by plateauing. In addition, there was no significant difference in percent coverage of blood vessels by AQP4+ end-feet in GM compared with cortex and WM for premature infants 23-34 wk GA. Our findings appear to be consistent with rat experiments, in which Western blot analysis of membrane fraction of whole brain showed no labeling for AQP4 at 14-21 d gestation, weak labeling at postnatal d 1,2\% of adult level at postnatal d 7, and 63\% at postnatal d 28 (28). AQP4 is viewed as the prime mediator of transmembrane transport in CNS and is strategically located predominantly in astrocyte end-feet and ependyma to promote water exchange between 

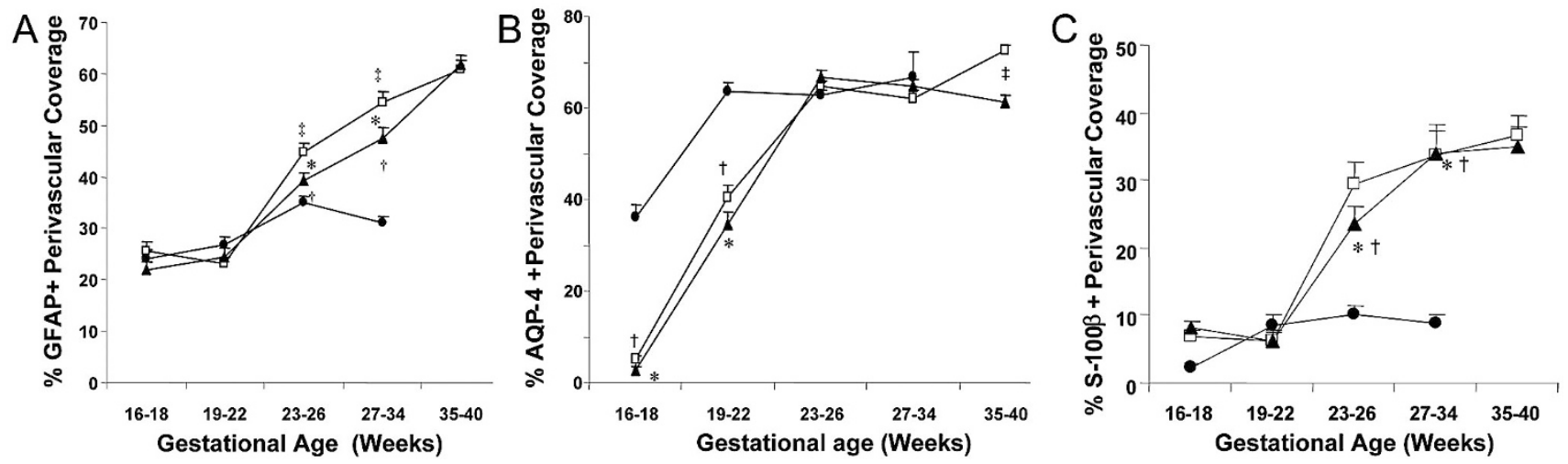

Figure 6. (A) Decreased perivascular coverage by GFAP+ end-feet in GM compared with cortex and WM in preterm infants (mean \pm SEM). End-feet perivascular coverage increased in cortex and WM with GA from 19 to $40 \mathrm{wk}(p<0.001)$. In contrast, in GM, GFAP+ end-feet increased only between 19-22 and 23-26 wk $(p<0.05)$, without significant change during other periods of gestation. Coverage by GFAP+ end-feet was decreased in GM, compared with cortex and WM, in premature infants. Coverage was greater in WM than in cortex for 23-27 and 27-34 wk. (B) Quantification of perivascular coverage by AQP4+ end-feet in GM, cortex, and WM. Vascular coverage by AQP4+ end-feet in GM increased significantly from $36 \%$ to $63.7 \%$ at $19-22$ wk $(p<0.001$ ), then plateaued. In cortex and WM, AQP4+ end-feet coverage was $<5 \%$ at $16-18 \mathrm{wk}$, increased consistently to about $63 \%$ by $23-27 \mathrm{wk}(p<0.001)$, then plateaued. Percentage AQP4+ end-feet coverage was significantly greater in GM than cortex and WM in fetuses $16-22$ wk $(p<0.001)$. In premature infants (23-34 wk), there was no significant difference in AQP4+ end-feet coverage in GM compared with cortex and WM. In term infants, percentage coverage was greater in WM than in cortex $(p<0.001)$. (C) Quantification of perivascular coverage by S-100 $\beta+$ end-feet in GM, cortex, and WM. S-100 $\beta+$ perivascular coverage increased in GM between 16-18 and 19-22 wk $(p<0.05)$, but no significant change was observed thereafter. However, in cortex and WM, perivascular coverage consistently increased with GA from 22 to $40 \mathrm{wk}(p<0.001)$ without change between 16 and 22 wk. Coverage by S-100 $\beta+$ end-feet was decreased in GM compared with cortex and WM for premature infants 23-34 wk $(p<0.001)$. In term infants, perivascular coverage was about 35\% without difference between cortex and WM. * $p<0.001 \mathrm{GM} v s \mathrm{WM} ;{ }^{\dagger} p<0.05 \mathrm{GM} v s$ cortex; ${ }^{*} p<0.05$ cortex $v s$ WM. Closed circles (——) denote GM, open squares (— $\square$-) denote white matter, and closed triangles $(-\mathbf{\Delta}-)$ denote cortex.

blood and CSF (29). AQP4 plays a role in pathophysiologic conditions, as shown by reduced edema formation after water intoxication and after focal cerebral ischemia in AQP4+ knock-out mice (30). Hence, our finding of no significant difference in $\mathrm{AQP} 4+$ end-feet in premature and mature infants 23-40 wk GA suggests that preterm and term infants may be at equal risk of brain edema in pathologic conditions. In addition, early development of AQP4 molecules points toward the existence of a close relationship between water transport regulation and brain development.

The pathogenesis of GMH-IVH is not well understood. However, our finding of decreased expression of the cytoskeletal protein GFAP in astrocyte end-feet along the vasculature of the GM compared with cortex or WM for premature infants 23-34 wk GA is consistent with a cytostructural difference that may ultimately prove responsible for the fragility of the GM vasculature. Additional investigation in the animal model of IVH involving manipulation of GFAP level is needed to further confirm the role of GFAP expression in astrocyte end-feet to provide structural integrity to the GM vasculature and prevent GMH-IVH. Furthermore, an evaluation of other components of the BBB including pericytes and basal lamina may also unravel the mysteries related to the GM vascular fragility.

Acknowledgments. The authors thank Joane Abrahams for her technical help with images.

\section{REFERENCES}

1. Ballabh P, Braun A, Nedergaard M 2004 The blood-brain barrier: an overview: structure, regulation, and clinical implications. Neurobiol Dis 16:1-13
2. Volpe JJ 1997 Brain injury in the premature infant. Neuropathology, clinical aspects, pathogenesis, and prevention. Clin Perinatol 24:567-587

3. Ballabh P, Braun A, Nedergaard M 2004 Anatomic analysis of blood vessels in germinal matrix, cerebral cortex, and white matter in developing infants. Pediatr Res $56: 117-124$

4. Ballabh P, Hu F, Kumarasiri M, Braun A, Nedergaard M 2005 Development of tight joint molecules in blood vessels of germinal matrix, cerebral cortex and white matter. Pediatr Res 58:791-798

5. Bass T, Singer G, Slusser J, Liuzzi FJ 1992 Radial glial interaction with cerebral germinal matrix capillaries in the fetal baboon. Exp Neurol 118:126-132

6. Ment LR, Stewart WB, Ardito TA, Madri JA 1995 Germinal matrix microvascular maturation correlates inversely with the risk period for neonatal intraventricular hemorrhage. Brain Res Dev Brain Res 84:142-149

7. Bertossi M, Virgintino D, Errede M, Roncali L 1999 Immunohistochemical and ultrastructural characterization of cortical plate microvasculature in the human fetus telencephalon. Microvasc Res 58:49-61

8. Ambrosi G, Virgintino D, Benagiano V, Maiorano E, Bertossi M, Roncali L 1995 Glial cells and blood-brain barrier in the human cerebral cortex. Ital J Anat Embryol 100 (suppl 1):177-184

9. Wilkinson M, Hume R, Strange R, Bell JE 1990 Glial and neuronal differentiation in the human fetal brain 9-23 weeks of gestation. Neuropathol Appl Neurobiol 16:193-204

10. Amiry-Moghaddam M, Xue R, Haug FM, Neely JD, Bhardwaj A, Agre P, Adams ME, Froehner SC, Mori S, Ottersen OP 2004 Alpha-syntrophin deletion removes the perivascular but not endothelial pool of aquaporin-4 at the blood-brain barrier and delays the development of brain edema in an experimental model of acute hyponatremia. FASEB J 18:542-544

11. Simard M, Arcuino G, Takano T, Liu QS, Nedergaard M 2003 Signaling at the gliovascular interface. J Neurosci 23:9254-9262

12. Boyes BE, Kim SU, Lee V, Sung SC 1986 Immunohistochemical co-localization of S-100b and the glial fibrillary acidic protein in rat brain. Neuroscience 17:857-865

13. Eng LF, Ghirnikar RS, Lee YL 2000 Glial fibrillary acidic protein GFAP-thirtyone years (1969-2000). Neurochem Res 25:1439-1451

14. Nielsen S, Nagelhus EA, Amiry-Moghaddam M, Bourque C, Agre P, Ottersen OP 1997 Specialized membrane domains for water transport in glial cells: highresolution immunogold cytochemistry of aquaporin-4 in rat brain. J Neurosci 17:171-180

15. Debus E, Weber K, Osborn M 1983 Monoclonal antibodies specific for glial fibrillary acidic (GFA) protein and for each of the neurofilament triplet polypeptides. Differentiation 25:193-203

16. Kacem K, Lacombe P, Seylaz J, Bonvento G 1998 Structural organization of the perivascular astrocyte endfeet and their relationship with endothelial glucose transporter: a confocal microscopy study. Glia 23:1-10

17. Herrmann H, Aebi $U 2000$ Intermediate filaments and their associates: multi-talented structural elements specifying cytoarchitecture and cytodynamics. Curr Opin Cell Biol 12:79-90 
18. Liedtke W, Edelmann W, Bieri PL, Chiu FC, Cowan NJ, Kucherlapati R, Raine CS 1996 GFAP is necessary for the integrity of CNS white matter architecture and long-term maintenance of myelination. Neuron 17:607-615

19. Ciesielski-Treska J, Bader MF, Aunis D 1982 Microtubular organization in flat epitheloid and stellate process-bearing astrocytes in culture. Neurochem Res 7:275286

20. Trimmer PA, Reier PJ, Oh TH, Eng LF 1982 An ultrastructural and immunocytochemical study of astrocytic differentiation in vitro: changes in the composition and distribution of the cellular cytoskeleton. J Neuroimmunol 2:235-260

21. Ribotta MG, Menet V, Privat A 2004 Glial scar and axonal regeneration in the CNS: lessons from GFAP and vimentin transgenic mice. Acta Neurochir Suppl 89:87-92

22. Melcangi RC, Magnaghi V, Cavarretta I, Riva MA, Martini L 1997 Corticosteroid effects on gene expression of myelin basic protein in oligodendrocytes and of glial fibrillary acidic protein in type 1 astrocytes. J Neuroendocrinol 9:729-733

23. Morrison RS, De Vellis J, Lee YL, Bradshaw RA, Eng LF 1985 Hormones and growth factors induce the synthesis of glial fibrillary acidic protein in rat brain astrocytes. J Neurosci Res 14:167-176
24. [No authors listed] 1995 Effect of corticosteroids for fetal maturation on perinatal outcomes. NIH Consensus Development Panel on the Effect of Corticosteroids for Fetal Maturation on Perinatal Outcomes. JAMA 273:413-418.

25. Sasaki A, Hirato J, Nakazato Y, Ishida Y 1988 Immunohistochemical study of the early human fetal brain. Acta Neuropathol (Berl) 76:128-134

26. Gould SJ, Howard S 1987 An immunohistochemical study of germinal layer in the late gestation human fetal brain. Neuropathol Appl Neurobiol 13:421-437

27. Sarnat HB 1992 Regional differentiation of the human fetal ependyma: immunocytochemical markers. J Neuropathol Exp Neurol 51:58-75

28. Wen H, Nagelhus EA, Amiry-Moghaddam M, Agre P, Ottersen OP, Nielsen S 1999 Ontogeny of water transport in rat brain: postnatal expression of the aquaporin- 4 water channel. Eur J Neurosci 11:935-945

29. Badaut J, Lasbennes F, Magistretti PJ, Regli L 2002 Aquaporins in brain: distribution, physiology, and pathophysiology. J Cereb Blood Flow Metab 22:367-378

30. Verbavatz JM, Ma T, Gobin R, Verkman AS 1997 Absence of orthogonal arrays in kidney, brain and muscle from transgenic knockout mice lacking water channel aquaporin-4. J Cell Sci 110:2855-2860 